\title{
Exploration in 3D Multimodal Virtual Environment for Nonvisual Spatial Recognition
}

\author{
Ying Ying Huang \\ HCI Dept. School of Computer Science and Communication, \\ Royal Institute of Technology \\ SE-10044, Stockholm, Sweden \\ yingying@csc.kth.se
}

\begin{abstract}
Visually impaired people have more problems on navigation both indoors and outdoors compairing with sighted people because of lacking the visual channel. Appropriate learning tools about spatial information could be used as a preparation for navigation before going to the real place. In this paper, a study on exploring access in non-visual spatial information by visually impaired people and the navigation and orientation with haptic and audio cues is presented. A 3D virtual simulation prototype of a real world environment was created for this purpose. Different navigation tools were designed in the prototype with haptic and audio cues. The main results from qualitative analysis of the study reported are: (a) the development of a virtual 3D environment prototype; (b) whether the spatial information for establishing the mental mapping of the space could be acquired by using touch and hearing channels ; (c) how haptic and audio cues can facilitate the navigation, mobility and orientation in such $3 \mathrm{D}$ virtual environments.
\end{abstract}

Keywords: Haptic, Audio, Virtual Reality, Navigation, Cognitive Mapping.

\section{Introduction}

Spatial information presented visually is not easily accessible for visually impaired users. Current access technologies, such as screen readers, can not convey spatial layout or structure. Virtual reality has been a popular paradigm in simulation based training, in the gaming and entertainment industries. It has also been used for rehabilitation and learning environments for people with sensory, physical, mental, and learning disabilities [1] 2]. Particularly haptic technology offers alternative means of presenting spatial information to visually disabled users. Research on the implementation of haptic technologies within Virtual Environments (VEs) has reported on potential for supporting development of cognitive models of navigation and spatial knowledge with sighted people [3] [4] [5] [6] and blind people [7] 8]. However, users normally interact with the virtual world using a single "pen" probe or stylus. It is not very easy for them to interact with $3 \mathrm{D}$ models in the absence of visual cues because of the point-contact nature of 
the haptic device. There are two main problems as mentioned in the study by Walker et al. [10] One is that it can be hard to locate the data initially. The second is that it will be difficult to maintain contact with it after locating an object. As Sjostrom [5] reports: "For a blind person, locating an object with a point probe can be as hard as finding a needle in a haystack". Colwell et al. 9] describe users as becoming "lost in haptic space" when they are unfamiliar with this type of interaction, as they frequently lose contact with an object they are trying to explore.

A 3D haptic and audio virtual simulation environment of Kulturhuset (House of Culture) in Stockholm was developed for this study. Such touch-and-hearing tools could potentially be used by visually impaired users to learn about physical spaces that they are required to navigate (e.g., school, work place, public buildings). The prototype allows users to explore the virtual Kulturhuset building to find different locations. The Research questions of this study were: (a) the development of a virtual 3D environment helping visually impaired users to learn about real space where they are required to navigate (e.g. in schools, work places, public buildings), (b) whether the spatial information for establishing the mental mapping of the space could be acquired by using compensatory sensorial channels (e.g., touch and hearing), as an alternative to the visual channel; (c) how haptic and audio cues can enable the efficient navigation, mobility and orientation in such 3D virtual environments. Results from qualitative analysis regarding learning process and actual performance in the 3D virtual world are presented.

\section{The Study}

Five visually impaired participants took part in the study, one female 23 years old, males 28, 35, 40 and 52 years old. The participants tried the Kulturhuset prototype (Fig. 1, Fig. 2) individually. Fig. 3 shows the setting of the study environment. Participants should do two tasks (objects identification) under each condition(Haptic visual/ haptic and audio and visual) in a random order. We keep the visual channel available. In all conditions there are magnetic forces

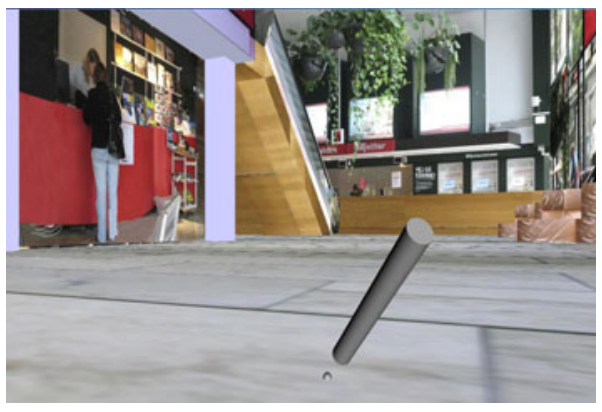

Fig. 1. A virtual version of Kulturhuset

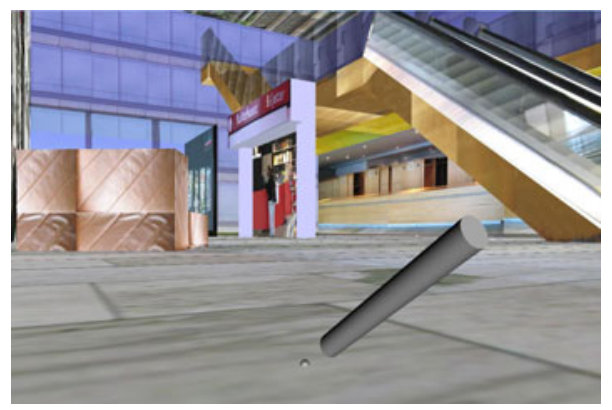

Fig. 2. Escalator 


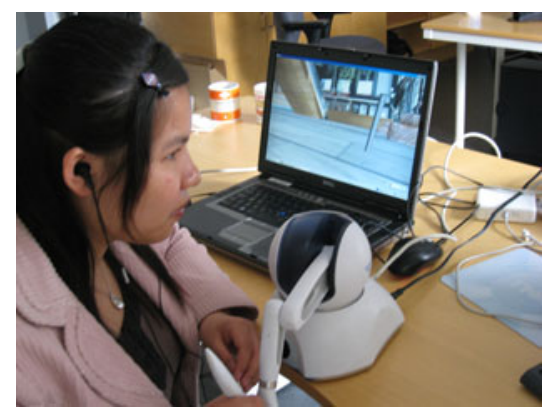

Fig. 3. User interacting with the haptic virtual environment using a PHANTOM Omni force feedback device (source: SensAble Technologies Inc)

designed for each object in order to attract the user when they move closer than 5 meters.

In the test, a Dell Precision Laptop with two dual core processors is used. One PHANTOM Omni, the haptic device, is connected serially to Laptop. For developing the application H3DAPI 2.0X3D, Python as well as Visual Studio .NET 2003, are employed. H3DAPI can be used both as an API to implement X3D applications, and as a loader of applications built in X3D and Python. A video camera is used for recording purpose and Camstudio for screen capturing during the tests. A video analysis was performed after the test sessions in this study. An interview was conducted for about 20 minutes after the test. Finally, the results with qualitative analysis were compared in order to obtain general findings.

\section{Result and Discussion}

This work presents an effort to explore the impacts of haptic and audio cues on non-visual spatial recognition for visually impaired users. Four issues were analyzed based the study, they are: the possibility to access a $3 \mathrm{D}$ virtual multimodal envrionment; Object Recognition; Spatial Recognition and the impacts of Haptic and Audio Aids on Navigation, Mobility and Orientation. The most important conclusions that can be drawn for the design of such environments are listed below:

- It is possible to access the virtual world of a real world by visually impaired users with haptic and audio cues. Both objects and spatial structure recognition will be faster, more accurate, and easier when both types of cues are available. - Haptic cues alone will aid structure recognition, but audio cues alone will not. Once a node has been located, audio cues will provide more efficient means (speech or non-speech) of identifying it than haptic cues. Therefore, participants will rate haptic cues as more useful for identifying structure and audio cues as more useful for identifying objects. 
- Integration of haptic and audio feedback provides more efficient navigation, mobility and orientation that either of them alone. It is better to have larger surfaces in all objects. The design of spatial architecture, fixed reference points like walls, floor, fixed objects and sensitive texture with haptic feedback are important to support orientation.

We hope that the study could be an investigation on the the possibility to access a 3D virtual multimodal envrionment for blind and visually impaired people to use haptic and audio virtual reality software for journey planning before they move into the real world. It could also serve as an input for visually impaired users to access online 3D virtual reality environments like Second Life with haptic and audio cues.

\section{References}

1. Schultheis, M.T., Rizzo, A.A.: The application of virtual reality technology for rehabilitation. Rehabilitation Psychology 46(3), 296-311 (2001)

2. Standen, P.J., Brown, D.J., Cromby, J.J.: The effective use of virtual environments in the education and rehabilitation of students with intellectual disabilities. British Journal of Education Technology 32(3), 289-299 (2001)

3. Hurmuzlu, Y., Ephanov, A., Stoianovici, D.: Effect of a Pneumatically Driven Haptic Interface on the Perceptional Capabilities of Human Operators. Teleoperators and Virtual Environments 7(3), 290-307 (1998)

4. Kuber, R., Yu, W., Mcallister, G.: Towards Developing Assistive Haptic Feedback for Visually Impaired Internet Users. In: Proceedings of the 2007 Conference on Human Factors in Computing Systems, San Jose, California, USA, pp. 1525-1534 (2007)

5. Sjostrom, C.: Designing Haptic Computer Interfaces for Blind People. In: Proc. ISSPA, vol. 1, pp. 68-71 (2001)

6. Bergamasco, M., Avizzano, C., Di Petri, G., Barbagli, F., Frisoli, A.: The museum of pure form: system architecture. In: Proceedings of 10th IEEE International Workshop on Robot and Human Interactive Communication, pp. 112-117. IEEE Press, Piscataway (2001)

7. Bergamasco, M., Prisco, G.: Design of an anthropomorphic haptic interface for the human arm. In: Shirai, Y., Hircse, S. (eds.) Robotic Research, the Eight International Symposiums, pp. 278-289. Springer, London (1998)

8. Frisoli, A., Simoncini, F., Bergamasco, M.: Mechanical design of a haptic interface for the hand. In: Proceedings of 2002 ASME DETC 27th Biennial Mechanisms and Robotics Conference, pp. 25-32. ASME Press, Montreal (2002)

9. Colwell, C., Petrie, H., Kornbrot, D.: Haptic virtual reality for blind computer users. Paper Presented at the Assets 1998 Conference, Los Angeles, CA (1998), http://phoenix.herts.ac.uk/sdru/pubs/VE/colwell.html

10. Walker, B.N., Lindsay, J.: Navigation performance with a virtual auditory display: Effects of beacon sound, capture radius, and practice. Human Factors 48(2), 265278 (2006) 